\title{
The manifestation of individual differences in sensitivity to punishment during resting state is modulated by eye state
}

\author{
Víctor Costumero ${ }^{1,2}$ • Jesús Adrián-Ventura ${ }^{2}$ Elisenda Bueichekú ${ }^{2} \cdot$ Anna Miró-Padilla $^{2}$. \\ María-Ángeles Palomar-García ${ }^{2}$ - Lidón Marin-Marin ${ }^{2}$ - Esteban Villar-Rodríguez ${ }^{2}$ Naiara Aguirre $^{2}$. \\ Alfonso Barrós-Loscertales ${ }^{2}$. César Ávila ${ }^{2}$
}

Accepted: 21 November 2020 / Published online: 12 January 2021

(C) The Psychonomic Society, Inc. 2021

\begin{abstract}
Structural and functional neuroimaging studies have shown that brain areas associated with fear and anxiety (defensive system areas) are modulated by individual differences in sensitivity to punishment (SP). However, little is known about how SP is related to brain functional connectivity and the factors that modulate this relationship. In this study, we investigated whether a simple methodological manipulation, such as performing a resting state with eyes open or eyes closed, can modulate the manifestation of individual differences in SP. To this end, we performed an exploratory fMRI resting state study in which a group of participants $(n=88)$ performed a resting state with eyes closed and another group $(n=56)$ performed a resting state with eyes open. All participants completed the Sensitivity to Punishment and Sensitivity to Reward Questionnaire. Seed-based functional connectivity analyses were performed in the amygdala, hippocampus, and periaqueductal gray (PAG). Our results showed that the relationship between SP and left amygdala-precuneus and left hippocampus-precuneus functional connectivity was modulated by eye state. Moreover, in the eyes open group, SP was negatively related to the functional connectivity between the PAG and amygdala and between the PAG and left hippocampus, and it was positively related to the functional connectivity between the amygdala and hippocampus. Together, our results may suggest underlying differences in the connectivity between anxietyrelated areas based on eye state, which in turn would affect the manifestation of individual differences in SP.
\end{abstract}

Keywords Sensitivity to punishment $\cdot$ Anxiety $\cdot$ Resting state $\cdot$ Amygdala $\cdot$ Hippocampus $\cdot$ Eyes open/closed

\section{Introduction}

Imagine a situation where something you are looking at is directly threatening your body integrity. Punishment anticipation increases as the threat approaches, but curiously, some people prefer to keep looking at what is actually happening (e.g., looking at the needle during a blood extraction), whereas others prefer to stop focusing on it after evaluating the situa-

Víctor Costumero and Jesús Adrián-Ventura contributed equally to this work.

Víctor Costumero

vcostume@uji.es

1 Center for Brain and Cognition, University Pompeu Fabra, Ramon Trias Fargas, 25-27 08005 Barcelona, Spain

2 Neuropsychology and Functional Neuroimaging Group, Jaume I University, Castellón, Spain tion. The reason for individual differences in coping with threat is still an unresolved question. However, the study of the neural systems underlying defensive behaviors, and the factors that modulate these systems, may contribute to understanding this phenomenon.

Fear and anxiety are adaptive emotions that engage responses to cope with actual and anticipated threat. The reinforcement sensitivity theory (RST) proposes that these emotions are mediated by two separate but interacting neurobiological systems involved in the defense of the organism (Gray \& McNaughton, 2000): the fight-flight-freeze system (FFFS) associated with fear, and the behavioral inhibition system (BIS) associated with anxiety. The FFFS mainly comprises the amygdala, medial hypothalamus, and periaqueductal gray (PAG), and it is activated whenever the goal is to remove danger. The BIS is mainly composed of the septohippocampal system and amygdala, and it is activated when there are conflicting goals, as in the case of having to approach a potential threat. The RST suggests that these defensive neural systems 
are modulated by individual differences in the intensity of the perceived threat, a dimension that is conceptualized as a personality trait of sensitivity to punishment (SP) (McNaughton $\&$ Corr, 2004). Thus, in the same situation, individuals with higher SP would generally perceive threatening stimuli as more intense. Therefore, high SP individuals are more prone to experiencing fear, worry, and rumination and showing avoidance and risk-assessment behaviors (Corr, 2004; McNaughton \& Corr, 2004). High SP has been proposed as a vulnerability factor for anxiety disorders (Gray \& McNaughton, 2000), and empirical studies have shown a positive relationship between measures of this trait and anxiety disorder symptoms (Bijttebier, Beck, Claes, \& Vandereycken, 2009).

In recent years, neuroimaging studies have contributed to our understanding of the brain regions mediating individual differences in SP. For example, MRI structural studies have related SP (or related traits) with the volume and cortical thickness of medial prefrontal areas, amygdala, and hippocampus (Adrián-Ventura, Costumero, Parcet, \& Ávila, 2019; Barrós-Loscertales et al., 2006; Cherbuin et al., 2008; Fuentes et al., 2012; Holmes et al., 2012; Levita et al., 2014). Furthermore, functional MRI studies have shown that SP (or related traits) is associated with the activity of the cingulate cortex, precuneus, and amygdala during processing of negative events (Kennis, Rademaker, \& Geuze, 2013). However, little is known about how individual differences in SP are related to brain functional connectivity. As far as we know, only one study has investigated individual differences in functional connectivity in defensive system areas using a specific scale to measure SP in the context of RST (Hahn et al., 2010). This study showed that the connectivity between the hippocampus and amygdala during the processing of cues indicating potential monetary loss was positively correlated with the punishment sensitivity trait. Other studies have investigated individual differences in functional connectivity during resting state ( $\mathrm{rsFC}$ ) using scales related to $\mathrm{SP}$, such as neuroticism from the NEO-PI (Adelstein et al., 2011; Aghajani et al., 2014; Kruschwitz et al., 2014), the Eysenck Personality Questionnaire (Y. Pang et al., 2016), and the ZuckermanKuhlman Personality Questionnaire (Gentili et al., 2017). Studies also used trait anxiety from the State-Trait Anxiety Inventory (Kim, Gee, Loucks, Davis, \& Whalen, 2011; Modi, Kumar, Kumar, \& Khushu, 2015) and harm avoidance measures (Baeken et al., 2014; Huggins, Belleau, Miskovich, Pedersen, \& Larson, 2018; Y. Li, Qin, Jiang, Zhang, \& Yu, 2012; Markett et al., 2013; Meylakh \& Henderson, 2016). Most of these studies investigated amygdala connectivity, showing that SP-related traits were positively correlated with the connectivity between this region and lateral occipital areas and fusiform gyrus and negatively correlated with the connectivity between amygdala and ventromedial prefrontal cortex, cuneus, insula, and temporal cortex areas (Aghajani et al.,
2014; Baeken et al., 2014; Gentili et al., 2017; Kim et al., 2011; Kruschwitz et al., 2014; Y. Li et al., 2012). However, a number of results were not replicated across studies, and in some cases, they were even contradictory. For example, the rsFC strength between the amygdala and inferior frontal cortex was positively correlated with harm avoidance in one study (Baeken et al., 2014) but negatively correlated with neuroticism in another (Gentili et al., 2017). Likewise, one study showed a positive relationship between neuroticism from the NEO-PI and the connectivity between the amygdala and precuneus (Aghajani et al., 2014), whereas others showed negative associations using other neuroticism scales (Gentili et al., 2017; Y. Pang et al., 2016). Psychometric studies have shown strong relationships between these scales (Aluja \& Blanch, 2011; Caseras, Àvila, \& Torrubia, 2003). From a neurobiological perspective, all of these personality traits are expected to share a similar biological substrate related to anxiety. Therefore, it is still necessary to gather more evidence to clarify the inconsistent or contradictory results found in these previous studies. Some studies have shed light on this issue by showing how specific factors, such as gene expression (Buckholtz et al., 2008) or gender (Li et al., 2012) modulate the relationships between SP-related traits and rsFC. In line with these studies, we investigated whether a simple methodological manipulation, such as performing a resting state fMRI (rs-fMRI) with eyes open (EO) or eyes closed (EC), can impact the manifestation of individual differences in the rsFC of defensive system areas.

Evidence of modulatory effects of open or closed eyes on brain activity has been shown using EEG since the beginning of the past century (Berger, 1929). In the field of fMRI, evidence suggests that volitional closing or opening of the eyes leads to two different configurations of brain activity and connectivity: one associated with an "interoceptive" state, with $\mathrm{EC}$, and the other associated with an "exteroceptive" state, with EO (Costumero, Bueichekú, Adrián-Ventura, \& Ávila, 2020; Hüfner et al., 2009; Liang et al., 2014; Marx et al., 2003, 2004; Song et al., 2015; Wang, Li, Xu, \& Ding, 2015; Wei et al., 2018; Wiesmann et al., 2006; Xu et al., 2014; Zhang et al., 2015). Neuroimaging task studies have related the "exteroceptive" state to activations in attentional and oculomotor systems (e.g., superior parietal gyrus, anterior insula, thalamus, and frontal eye fields) and the "interoceptive" state with activity in regions related to mental imagery and multisensory integration, such as the lateral occipital areas, auditory cortex, postcentral gyrus, and medial frontal cortex (Marx et al., 2003, 2004; Wiesmann et al., 2006). Given these previous studies, we hypothesize that a different brain activity configuration in $\mathrm{EC}$ and $\mathrm{EO}$ conditions might lead to a different manifestation of individual differences in SP within these conditions because the behaviors that characterize SP involve both "interoceptive" (e.g., worry, rumination) and "exteroceptive" (e.g., threat detection, escape/avoidance) states. Supporting this 
hypothesis, previous studies showed that eye state modulated the relationship between high-frequency and low-frequency EEG rhythms and the neuroticism trait from the Eysenck Personality Questionnaire (Konareva, 2011a, b). Furthermore, fMRI studies have shown that blood oxygenlevel dependent (BOLD) signals of key regions within the defensive system, such as the hippocampus and amygdala, are modulated by EO and EC conditions (Ben-Simon, Podlipsky, Arieli, Zhdanov, \& Hendler, 2008; Jao et al., 2013; Liu, Dong, Zuo, Wang, \& Zang, 2013; Wiesmann et al., 2006). Moreover, modulatory effects of eye state have been shown in studies investigating individuals with generalized anxiety disorder (GAD). Thus, one fMRI study showed reduced rsFC between the dorsolateral prefrontal cortex and default mode network areas in individuals with GAD compared with controls, in the EC condition, but not in the EO condition (Li et al., 2016). Furthermore, an EEG study found that controls, but not GAD individuals, showed a significantly higher amplitude of heartbeat-evoked brain potential under EC than under EO (Pang et al., 2019).

In summary, the present study investigated the effects of the EO and EC conditions on the manifestation of SP-related individual differences in the rsFC of defensive system areas. Given that no previous research has examined such relationship, this study should be considered exploratory in nature. Even so, we expect to find differences in the relationship between SP and the rsFC of defensive system areas according to the EO and EC conditions. Specifically, we hypothesized that rsFC individual differences in interoceptive-related areas would be shown during EC, and individual differences in exteroceptive-related areas would be shown during EO. By considering the role of eye state during resting state, we attempt to provide new insights into the study of the neural basis of SP.

\section{Method}

\section{Participants}

A dataset consisting of 198 individuals ( 95 women; age: mean $=22.2$, standard deviation $[\mathrm{SD}]=4.3$, range $=18-40$ years) was collected from various projects performed by our research group using the same scanner. After subject exclusion due to excessive head motion (see preprocessing section), the final sample for analysis included 144 participants ( 75 women; age: mean $=22.0, \mathrm{SD}=4.0$, range $=40-18)$. Of them, 56 performed an EO resting state session, and 88 performed an EC resting state session. Demographic characteristics of these groups are reported in Table 1. All participants were righthanded according to the Edinburgh Handedness Inventory (Oldfield, 1971). No participant had a history of head injury with loss of consciousness, none currently used psychoactive medications, and none had ever been diagnosed with DSM-IV Axis I or II disorders or severe medical or neurological illnesses. Participants were informed of the nature of the research and provided written, informed consent before their participation in the study. All study procedures were approved by the Ethical Committee of the Jaume I University.

\section{Personality assessment}

The SP scale from the Sensitivity to Punishment and Sensitivity to Reward Questionnaire (SPSRQ) (Torrubia, Ávila, Moltó, \& Caseras, 2001) was used as a measure of the punishment sensitivity trait. Descriptive statistics for the personality measures in each group are reported in Table 1. The SP scale has good content validity and strongly correlates with other measures of punishment sensitivity, such as the Behavioral Inhibition Scale, Harm Avoidance, Punishment Expectancies, and anxiety scales (Caseras et al., 2003).

\section{Image acquisition}

Scan sessions required participants to be in a resting state. For the EC sessions, participants were instructed to simply rest with their eyes closed and not sleep or think about anything in particular. These same instructions, but with the specification of keeping their eyes open, were provided in the EO sessions. Immediately after scanning, participants were explicitly asked if they had followed the instructions and whether they had experienced any issues during the scan. None of the participants reported issues, and all of them confirmed that they had followed the instructions. Images were acquired on a 1.5T scanner (Siemens Avanto; Erlangen, Germany). Participants were placed in a supine position in the MRI scanner, and their heads were immobilized with cushions to reduce head motion. For the rs-fMRI, a total of 200 volumes were recorded using a gradient-echo $\mathrm{T} 2 *$-weighted echo-planar imaging sequence (TR, $2000 \mathrm{~ms}$; TE, $48 \mathrm{~ms}$; matrix, 64 x 64; voxel size, $3.5 \times 3.5 \mathrm{~mm}$; flip angle, $90^{\circ}$; slice thickness, 4 $\mathrm{mm}$; slice gap, $0.8 \mathrm{~mm}$ ). We acquired 24 interleaved axial slices parallel to the anterior-posterior commissure plane covering the entire brain. Before the rs-fMRI sequences, structural images were acquired using a high-resolution T1-weighted MPRAGE sequence with TR $=2200 \mathrm{~ms}$, TE $=3.79 \mathrm{~ms}$, TI $=1090 \mathrm{~ms}$, flip angle $15^{\circ}$, voxel size $=1 \times 1 \times 1 \mathrm{~mm}$, matrix $=$ $256 \times 256$, bandwidth $=160 \mathrm{hz} / \mathrm{px}$, which facilitated the localization and co-registration of the functional data.

\section{Image preprocessing}

We used Data Processing \& Analysis for Brain Imaging (DPABI V2.1, http://rfmri.org/dpabi) (Yan, Wang, Zuo, \& Zang, 2016) to perform rs-fMRI data processing. Preprocessing included the following steps: 1) removal of 
Table 1 Demographic characteristics, personality, and motion-related variables

\begin{tabular}{|c|c|c|c|}
\hline & Eyes closed $(\mathrm{N}=88)$ & Eyes open $(\mathrm{N}=56)$ & Differences \\
\hline \multicolumn{4}{|l|}{ Sociodemographic variables } \\
\hline Age & $22.4 \pm 4.8(18-40)$ & $21.3 \pm 2.1(18-28)$ & $\begin{array}{l}\mathrm{t}_{(129)}=1.83 \\
p=0.07\end{array}$ \\
\hline Gender & $\begin{array}{l}\text { Male: } 45.5 \% \\
\text { Female: } 54.5 \%\end{array}$ & $\begin{array}{l}\text { Male: } 51.8 \% \\
\text { Female: } 48.2 \%\end{array}$ & $\begin{array}{l}\chi_{(1)}^{2}=0.55 \\
p>0.1\end{array}$ \\
\hline Education level & $\begin{array}{l}\text { Basic: } 2.3 \% \\
\text { Middle: } 25 \% \\
\text { Superior: } 72.7 \%\end{array}$ & $\begin{array}{l}\text { Basic: } 0 \% \\
\text { Middle: } 14.3 \% \\
\text { Superior: } 85.7 \%\end{array}$ & $\begin{array}{l}\chi_{(2)}^{2}=3.9 \\
p>0.1\end{array}$ \\
\hline \multicolumn{4}{|l|}{ Personality } \\
\hline Sensitivity to Punishment & $10 \pm 5.3(0-23)$ & $8.7 \pm 4.4(1-21)$ & $\begin{array}{l}\mathrm{t}_{(142)}=1.56 \\
p>0.1\end{array}$ \\
\hline Sensitivity to Reward & $10.3 \pm 4.8(1-21)$ & $10.3 \pm 4.5(3-23)$ & $\begin{array}{l}\mathrm{t}_{(142)}=-0.22 \\
p>0.1\end{array}$ \\
\hline \multicolumn{4}{|l|}{ Motion-related variables } \\
\hline mean RMS & $0.108 \pm 0.04(0.04-0.26)$ & $0.108 \pm 0.04(0.06-0.23)$ & $\begin{array}{l}\mathrm{t}_{(142)}=-0.28 \\
p>0.1\end{array}$ \\
\hline mean FD Power & $0.123 \pm 0.03(0.05-0.2)$ & $0.113 \pm 0.03(0.06-0.2)$ & $\begin{array}{l}\mathrm{t}_{(142)}=1.73 \\
p=0.09\end{array}$ \\
\hline mean FD Jenkinson & $0.065 \pm 0.02(0.03-0.1)$ & $0.059 \pm 0.02(0.03-0.1)$ & $\begin{array}{l}\mathrm{t}_{(142)}=1.80 \\
p=0.08\end{array}$ \\
\hline mean FD Van Dijk & $0.028 \pm 0.01(0.01-0.07)$ & $0.025 \pm 0.01(0.01-0.05)$ & $\begin{array}{l}\mathrm{t}_{(142)}=1.31 \\
p>0.1\end{array}$ \\
\hline Volumes with FD Power $>0.2$ & $20.3 \pm 14.5(0-48)$ & $15.1 \pm 12(1-41)$ & $\begin{array}{l}\mathrm{t}_{(132)}=2.36 \\
p=0.02\end{array}$ \\
\hline
\end{tabular}

Descriptive statistics for quantitative variables show mean \pm standard deviation (min - max). For two sample $t$ test comparisons, Welch's correction was applied when the homoscedasticity assumption was not satisfied due to a rejection of the null hypothesis of equal variances using the Levene test $(p<$ 0.05). RMS = root mean squared; FD = Framewise Displacement.

the first five volumes; 2) slice timing correction; 3) head motion correction using a six-parameter (rigid body) linear transformation; 4) co-registration of the individual structural images to the mean functional image; 5) segmentation of structural images using the DARTEL tool (Ashburner, 2007); 6) removal of spurious variance through linear regression: 24 parameters from the head motion correction (Friston, Williams, Howard, Frackowiak, \& Turner, 1996), spike regression of volumes with framewise displacement $(\mathrm{FD})>0$. 2 mm (Power, Barnes, Snyder, Schlaggar, \& Petersen, 2012), white matter signal, cerebrospinal fluid signal, linear trends, and quadratic trends; 7) spatial normalization to the Montreal Neurological Institute (MNI) space (voxel size $3 \times 3 \times 3 \mathrm{~mm}$ ); 8) spatial smoothing ( $4 \mathrm{~mm})$; and 9) band-pass temporal filtering $(0.01-0.1 \mathrm{~Hz})$.

Participants with more than $1 \mathrm{~mm}$ or 1 degree of movement in any of the six directions, or less than 150 volumes with FD $<0.2 \mathrm{~mm}$ (ensuring at least 5 minutes of rest with low FD), were excluded from the analyses. Head motion measures for each group are reported in Table 1. Given that we found between-group differences in the number of volumes with FD Power $>0.2$, we included this variable as a nuisance regressor in our analyses.

\section{Functional connectivity analysis}

A seed-based correlation approach was performed to investigate how eye state modulates individual differences in the rsFC of the defensive system. In this method, the connectivity strength relies on the correlation between the averaged BOLD signal of a region of interest (ROI), also called the seed, and the BOLD signals from other parts of the brain (voxels or other ROIs). For this study, we defined ROIs for three key regions of the defensive system proposed in the most recent update of the RST (Gray \& McNaughton, 2000): the periaqueductal gray, the amygdala, and the hippocampus. The medial hypothalamus was not defined as a ROI, because susceptibility artifacts affected this region in our sample. Amygdala and hippocampus seeds (left and right, separately) were defined from the masks provided in the anatomical automatic labeling (AAL) atlas (Tzourio-Mazoyer et al., 2002). The PAG seed was defined from the mask provided by the Harvard Ascending Arousal Network (AAN) Atlas (Edlow et al., 2012). Pearson's correlation coefficient was used as a measure of rsFC. We performed seed-to-voxel and seed-toseed analyses. In seed-to-voxel analysis, a rsFC spatial map for each participant and seed was calculated by correlating the seed's time series with the time series of every other voxel in the brain. 
In seed-to-seed analysis, pairwise correlations between the time series of the defined seeds were performed. Fisher's $r$ to $z$ transformation was performed to normalize correlation values.

Between group comparisons for seed-to-voxel analyses were performed using Statistical Parametric Mapping 12 (SPM12, http://www.fil.ion.ucl.ac.uk/spm) and Matlab R2014b (MathWorks, Inc., Natick, MA). In order to study differences between the EC and EO groups in the association between SP and the rsFC maps, a whole brain interaction analysis was performed using GLM for each seed. The model included two regressors defining the groups and two regressors defining the SP scores (one per group). Age, sex, and the number of volumes with FD Power $>0.2$ also were included as nuisance regressors. The comparison of the regression slopes for SP regressors between groups was the contrast of interest. Given the nondirectional nature of our hypothesis, we used an F test in SPM to study between-group comparisons. Significance was determined using cluster-extent based thresholding at $p<0.05$ FDR-corrected, using a voxel-level primary threshold of $p<0$. 001 uncorrected. For seed-to-seed analyses, we performed the same interaction model as described above in SPSS 23 (IBM Corp.). The statistical threshold for this analysis was set at $p<$ 0.05 FDR-corrected (Benjamini \& Hochberg, 1995). For all significant interactions, we performed post hoc testing to further study the directionality of the results. For seed-to-voxel analyses, the post hoc tests consisted of multiple regression designs, including SP as the variable of interest for each group separately. These analyses were restricted to a mask of the voxels that showed a significant interaction effect in the between-group comparisons, with a threshold of $p<0.05$ FWE corrected at the voxel level. Given that the definition of nonindependent functional masks could bias the results, these analyses should be interpreted as merely descriptive. Post-hoc tests in seed-toseed analyses consisted of Pearson correlations between rsFC and SP for each group separately.

Finally, given the unbalanced number of participants in the EC and EO groups, and the fact that these groups differ or slightly differ on some variables (Table 1), we replicated all the analyses using matched groups. Specifically, we selected a subsample of 56 participants from the pool of 88 EC participants. This subsample was selected to match them exactly with the 56 participants from the EO group on sex and education level, showing minimal differences in age, sensitivity to punishment, number of volumes with FD Power $>0.2$, and mean FD Power (see supplementary Table 1). The results using these matched groups are reported in supplementary Table 2.

\section{Results}

Seed-to-voxel analysis was performed in order to study whole-brain differences between resting modalities in the relationship between SP and the rsFC maps. This analysis showed that SP-related individual differences in the $\mathrm{rsFC}$ maps of the amygdala and hippocampus were modulated by eye status. On the one hand, we found a significant interaction effect in the rsFC between the left amygdala and precuneus (Table 2). As Fig. 1a shows, the EC group showed a positive relationship between SP and the rsFC of the left amygdala with the precuneus. In contrast, the EO group showed a negative relationship between these variables. This shift in the relationship between SP and rsFC was confirmed in post hoc testing, which showed voxels with a significant positive

Table 2 Summary of the seed-to-voxel and seed-to-seed results

Functional connectivity methods and results

Seed-to-voxel analyses

Eyes closed vs. eyes open

\begin{tabular}{|c|c|c|c|c|c|c|c|}
\hline Seed & Cluster size & $\mathrm{F}$ & $p$ FDR corrected & \multicolumn{2}{|c|}{ MNI coordinates } & \multicolumn{2}{|c|}{ Anatomical area } \\
\hline Amygdala left & 31 & 21.66 & 0.002 & \multicolumn{2}{|c|}{$-9-5748$} & \multicolumn{2}{|c|}{ Precuneus } \\
\hline Hippocampus left & 59 & 28.58 & $<0.001$ & \multicolumn{2}{|c|}{$3-4254$} & \multicolumn{2}{|c|}{ Precuneus } \\
\hline \multicolumn{8}{|c|}{ Seed-to-seed analyses } \\
\hline & & \multicolumn{2}{|c|}{ Eyes closed vs. eyes open } & \multicolumn{2}{|c|}{ Eyes closed } & \multicolumn{2}{|c|}{ Eyes open } \\
\hline \multicolumn{2}{|l|}{ Seeds } & $\mathrm{t}$ & $p$ FDR corrected & $\mathrm{r}$ & $\mathrm{p}$ (unc.) & $\mathrm{r}$ & $\mathrm{p}$ (unc.) \\
\hline \multicolumn{2}{|c|}{ PAG - Amygdala left } & 3.51 & 0.004 & 0.08 & $>0.1$ & -0.39 & 0.003 \\
\hline \multicolumn{2}{|c|}{ PAG - Amygdala right } & 2.21 & 0.035 & 0.01 & $>0.1$ & -0.30 & 0.025 \\
\hline \multicolumn{2}{|c|}{ PAG - Hippocampus left } & 2.93 & 0.012 & 0.03 & $>0.1$ & -0.36 & 0.006 \\
\hline \multicolumn{2}{|c|}{ Amygdala left - Hippocampus left } & -2.23 & 0.035 & -0.11 & $>0.1$ & 0.27 & 0.046 \\
\hline \multicolumn{2}{|c|}{ Amygdala right - Hippocampus right } & -2.47 & 0.030 & -0.03 & $>0.1$ & 0.36 & 0.006 \\
\hline
\end{tabular}

Results for the interaction between sensitivity to punishment and eyes closed vs. eyes open on the resting state functional connectivity are displayed. Rvalues represent the correlation between "seed-to-seed" connectivity values and sensitivity to punishment. PAG = Periaqueductal Gray. 
a

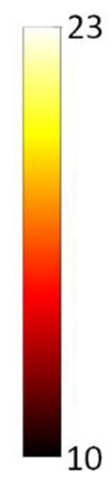

b

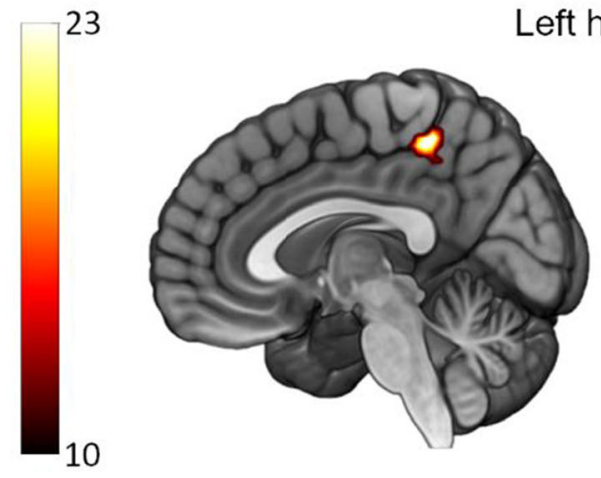

Fig. 1 Results from seed-to-voxel analysis. a Significant between-group differences in the relationship between sensitivity to punishment and individual differences in the functional connectivity of the amygdala. $\mathbf{b}$ Significant between-group differences in the relationship between sensitivity to punishment and individual differences in the functional connectivity of the hippocampus. The color bars represent the F-value applicable

relationship between $\mathrm{SP}$ and $\mathrm{rsFC}$ in the $\mathrm{EC}$ group $(\mathrm{k}=11)$ and voxels with a significant negative relationship between $\mathrm{SP}$ and $\mathrm{rsFC}$ in the EO group $(\mathrm{k}=15)$. Furthermore, we found a significant interaction effect in the rsFC between the left hippocampus and precuneus (Table 2). Similar to the results found in the left amygdala, SP was positively related to the rsFC between the left hippocampus and precuneus in the EC group but negatively related to it in the EO group (Fig. 1b). Again, these results were confirmed in the post hoc test performed in the EC group $(\mathrm{k}=14)$ and the EO group $(\mathrm{k}=36)$ separately. No significant results were found using the PAG seed in seed-to-voxel analyses. Similar results were found using the subsample of matched EC and EO participants (see supplementary Table 2).

Seed-to-seed analysis was performed to directly investigate how eye state modulates individual differences in the rsFC between our seeds (Table 2; Fig. 2). These analyses revealed a significant interaction effect in the rsFC between the PAG and left hippocampus. Post hoc analyses of this comparison revealed a significant negative correlation between $\mathrm{rsFC}$ and $\mathrm{SP}$ in the EO group, but not in the EC group. Furthermore, eye state modulated the rsFC between both the left and right

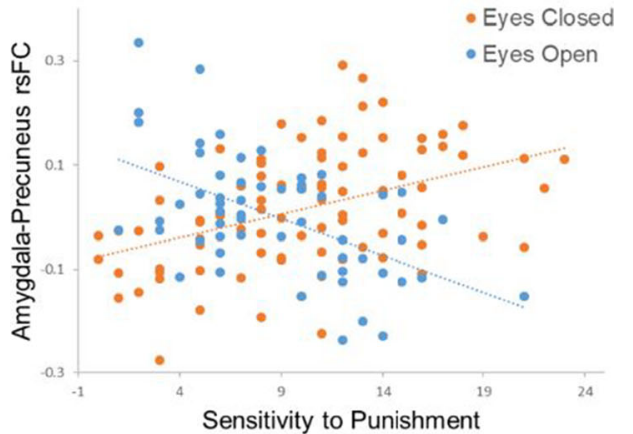

Sensitivity to Punishment 

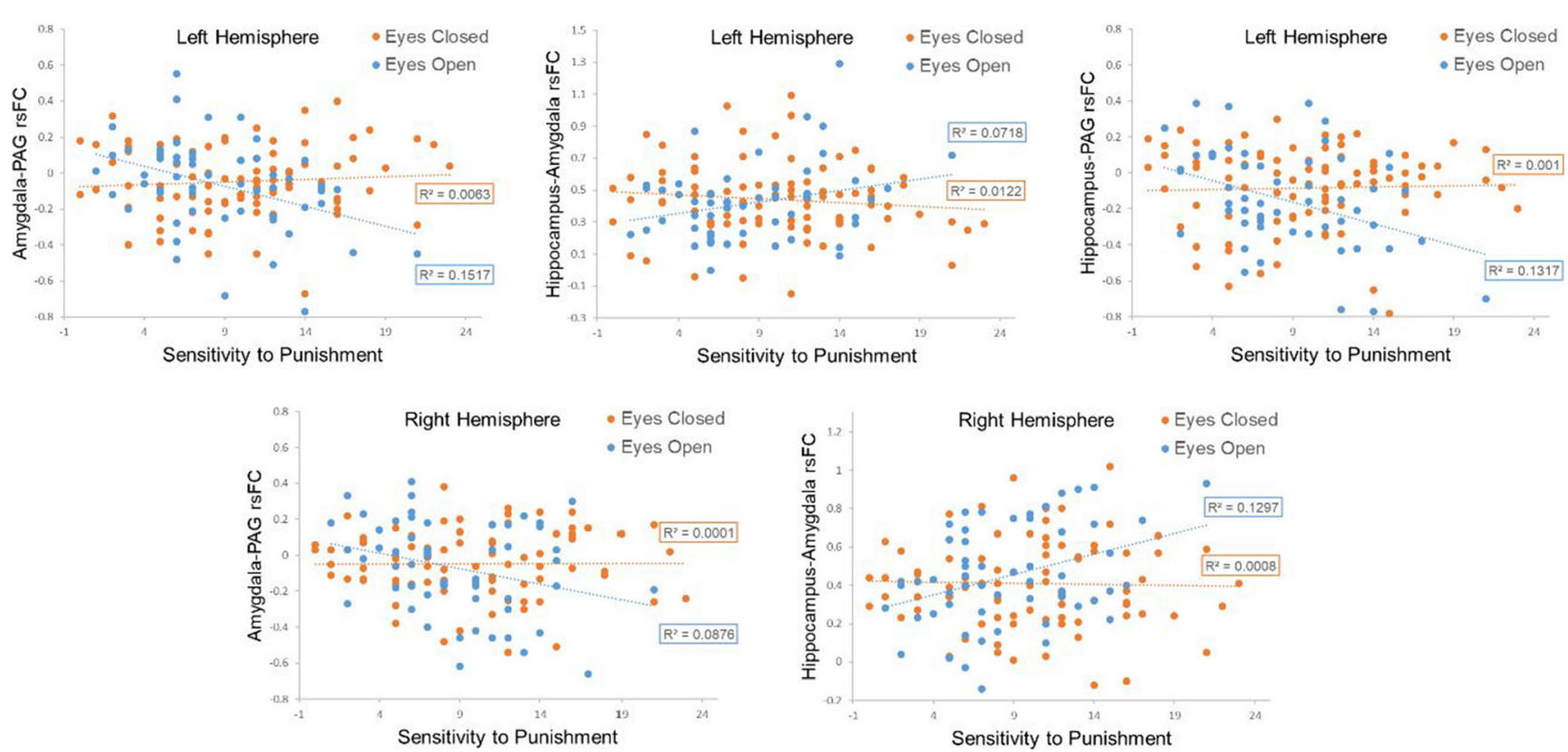

Fig. 2 Seed-to-seed results. Scatter plots show the relationship between sensitivity to punishment and resting state functional connectivity for each group separately.

defensive system areas. To achieve this, we compared two groups of participants: an rs-fMRI scan was performed on each participant, with either EO or EC, and the SP scale from the SPSRQ was also completed for each. Whole-brain voxelwise analyses showed that eye state modulates the relationship between SP and individual differences in the rsFC between the left amygdala and left hippocampus and the precuneus. Furthermore, seed-to-seed analysis showed that SP-related individual differences in the rsFC between the PAG and amygdala, the PAG and left hippocampus, and the amygdala and hippocampus were also modulated by eye state. These findings suggest underlying differences in the rsFC of anxietyrelated areas between $\mathrm{EC}$ and $\mathrm{EO}$, which may influence the manifestation of individual differences in SP.

Results of seed-to-voxel analysis showed a similar connectivity pattern in the left amygdala and left hippocampus seeds. With EC, the rsFC between the precuneus and these two regions was positively associated with SP. By contrast, SP was negatively associated with the rsFC between the precuneus and the two aforementioned regions during EO. The precuneus is functionally related to visuo-spatial imagery, episodic memory retrieval, self-processing, and consciousness (Cavanna \& Trimble, 2006). Although the precuneus is widely known for its involvement in the default mode network (Raichle et al., 2001), evidence from functional connectivity studies suggests that it could be subdivided based on its connectivity patterns in its dorsal and ventral portions (S. Zhang $\& \mathrm{Li}, 2012$ ). The ventral portion would be related to the default mode network, whereas the dorsal portion would be connected to areas associated with the dorsal attention network (Corbetta \& Shulman, 2002) - a brain network related to a variety of functions, including top-down control of visual attention, visuospatial imagery, and working memory (Corbetta, Patel, \& Shulman, 2008; Mellet, Petit, Mazoyer, Denis, \& Tzourio, 1998; Ptak, Schnider, \& Fellrath, 2017; Tomasino \& Gremese, 2016). In our study, the region of the precuneus that showed an interaction effect between eye state and SP in the connectivity with the amygdala and hippocampus was mainly located in the dorsal portion (Brodmann's area 7). The results of a recent study investigating functional connectivity differences between performance on a symbol digit modalities test and resting state suggest that this area might work as a transient in-between hub connecting the default mode network to task positive areas (da Silva, Rondinoni, \& Leoni, 2020). Specifically, this study showed that the dorsal precuneus was positively correlated with task positive regions, and negatively associated with the default mode network nodes when comparing task performance with the resting state condition. Furthermore, there is evidence suggesting that networks related to internally oriented and externally oriented cognition, such as default mode network, salience network, and dorsal attention network, dynamically switch between information processing modes as a function of eye state (Costumero et al., 2020; Xu et al., 2014; D. Zhang et al., 2015). Given these previous findings, our results might suggest that high SP is associated with a higher involvement of anxiety-related areas in the brain mechanisms implicated in orienting cognition toward internal or external stimuli. Individual differences in the connectivity between the amygdala and precuneus were previously shown in a study using the neuroticism scale from the NEO Five-Factor Inventory (Aghajani et al., 2014). Specifically, the authors of that study 
found that during an EC resting state session, the connectivity between the amygdala and dorsal precuneus was positively associated with individual differences in trait neuroticism. Therefore, the positive relationship between SP and the connectivity between the amygdala and precuneus in the EC group shown in our study converges with these findings.

When we analyzed seed-to-seed rsFC between our ROIs, we showed that eye state modulated individual differences in the PAG rsFC. Specifically, our results suggest that the rsFC of the PAG with the amygdala and left hippocampus is negatively associated with SP during EO. The PAG has mainly been related to the smallest defensive distances, such as fight, flight, and freeze (Fanselow, 1991). This region is structurally connected with the amygdala, and it shows functional connectivity with both the amygdala and hippocampus (Linnman, Moulton, Barmettler, Becerra, \& Borsook, 2012). The amygdala and hippocampus are suggested to be higher in the hierarchy of the defensive system, with the former implicated in the control of active avoidance and the arousal associated with anxiety, and the latter mainly related to anxiety (McNaughton \& Corr, 2008). Previous studies have shown modulatory effects of eye state in these three regions. Thus, the degree of rsFC between the PAG and the medial frontal cortex was associated with glutamate concentrations in the EC condition, but not in the EO condition (Duncan et al., 2013). Furthermore, previous evidence showed increased hippocampus activity after closing eyes in darkness (Wiesmann et al., 2006) and higher variance and regional homogeneity in amygdala BOLD signals in an EC condition compared with an EO condition (Jao et al., 2013; Liu et al., 2013). In our study, seed- to-seed analysis also showed differences in the relationship between SP and the rsFC between the amygdala and hippocampus as a function of eye state. SP-related individual differences in the rsFC between the hippocampus and amygdala were shown in a previous fMRI study investigating the brain response to punishment anticipation (Hahn et al., 2010). Specifically, in that study, the authors used the beta series correlation method to show that the connectivity between the hippocampus and amygdala during the presentation of visual cues signaling potential monetary loss was positively related to individual differences in SP. In our study, the significant interaction effects in the rsFC between the hippocampus and amygdala were driven by a positive association between SP and rsFC in the EO group, suggesting that individual differences in the connectivity between these regions are also present when eyes are open, but in the absence of punishment cues. Interestingly, Hahn et al. (2010) also performed a psychophysiological interaction analysis comparing punishment cues with neutral cues, but they did not find significant differences using this methodology. Psychophysiological interaction analysis is a method used to investigate specific differences in brain connectivity between task conditions. Given these negative results and the results presented here, future studies should determine whether the presence of aversive cues influences the relationship between SP and amygdala-hippocampus connectivity, or whether this relationship was actually driven by the existence of individual differences at rest. This distinction may imply a different brain predisposition related to SP; variances in connectivity observed in the presence of aversive cues would be

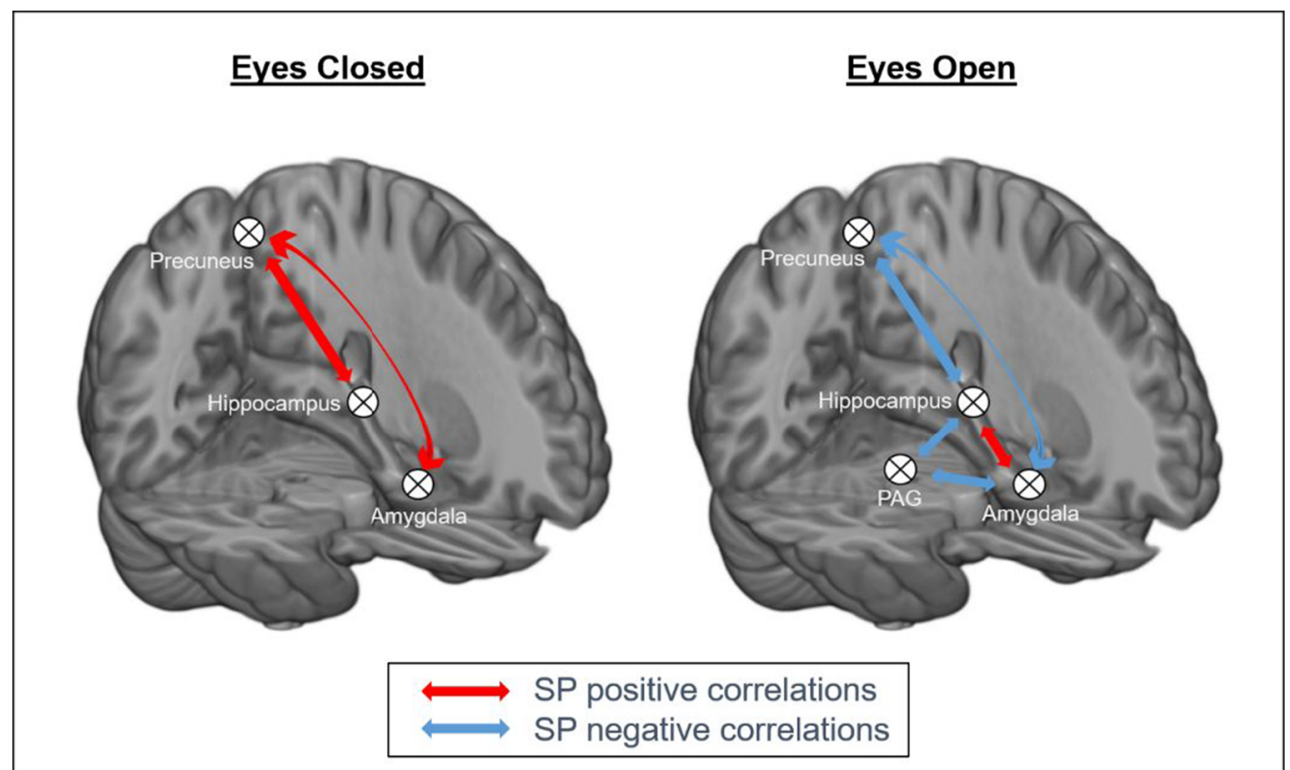

Fig. 3 Summary of the modulatory effects of eyes open and eyes closed conditions in the manifestation of individual differences in functional connectivity associated with sensitivity to punishment. Red arrows show the brain regions that present a significant positive relationship between functional connectivity and sensitivity to punishment in the eyes closed group (left) and the eyes open group (right), as determined by the post hoc tests. Blue arrows show the brain regions that present a significant negative relationship between functional connectivity and sensitivity to punishment in the eyes closed group (left) and the eyes open group (right), as determined by the post hoc tests. 
associated with individual differences in "control mode" (brain's response system to present stimuli), whereas variances in rsFC would be linked to differences in "checking mode" (brain activity in absence of stimuli; see Ávila \& Torrubia, 2008). Our results, thus, would suggest the latter.

There are several limitations and directions for future research that should be considered. First, we did not record specific measures of rumination, anxiety state, or personality measures other than the SPSRQ. Thus, interindividual variability in any of these dimensions could affect the observed between-group differences. Second, the amygdala, hippocampus, and PAG are not unitary regions and could be further subdivided into more specialized subareas. In this study, we used global ROIs as defined in anatomical atlases (Edlow et al., 2012; Tzourio-Mazoyer et al., 2002); however, the use of a different set of ROIs might provide results not observed in this study. Third, RST proposes cortical regions such as the prefrontal cortex or cingulate as part of the defensive system (McNaughton \& Corr, 2008). However, we did not include these regions in our study, because the large size of these areas limits their definition as discrete seeds, and the model is not specific about the possible subareas associated with SP. Finally, it could be worthwhile to study the possible implications of our results in specific psychopathologies, such as anxiety or mood disorders. For example, previous studies in depressed individuals have related rumination with increased activity in the amygdala, hippocampus, and precuneus (Burkhouse et al., 2017; Mandell, Siegle, Shutt, Feldmiller, $\&$ Thase, 2014), and self-reported rumination measures have been positively associated with the SP trait (Leen-Feldner, Zvolensky, Feldner, \& Lejuez, 2004). In our study, eye state modulated the relationship between SP and $\mathrm{rsFC}$ in these structures. Thus, the question arises about whether eye state might play a role in ruminative thinking. Furthermore, previous evidence has related GAD and excessive worry to an altered processing of interoceptive signals during conditions of external processing, such as EO resting or emotional stimuli processing (Pang et al., 2019; Weber-Goericke \& Muehlhan, 2019). Thus, the results of our study might have implications for therapeutic approaches performed within a specific eye state, such as mindfulness meditation; indeed, there is evidence suggesting that the success of this technique is modulated by personality factors (Ding, Tang, Deng, Tang, \& Posner, 2015).

In summary, in this study we have shown that opening or closing eyes during resting state modulates the relationship between SP and individual differences in the rsFC of defensive system areas. Specifically, our results showed that precuneus-amygdala and precuneus-hippocampus $\mathrm{rsFC}$ were positively related to SP during EC, but negatively during EO. Moreover, during EO, SP was positively related to the rsFC between the hippocampus and amygdala, and negatively related to the rsFC between the PAG and the amygdala and hippocampus. These findings converge with the existence of a differential brain information processing mode associated with exteroceptive and interoceptive states, and suggest that these states affect the manifestation of individual differences in SP. Therefore, eye state should be considered as a modulatory factor in future rsFC studies of anxiety.

Supplementary Information The online version contains supplementary material available at https://doi.org/10.3758/s13415-020-00856-8.

Acknowledgments This work was supported by grants from Generalitat Valenciana (PROMETEO/2017/109) and Ministerio de Economía y Competitividad (PSI2016-78805-R) to C.A. Also, it was supported by a grant from Jaume I University (UJI-B2016-21) to A.B-L. Additionally, this work was supported by a pre-doctoral graduate program grant (National FPU to J.A-V, L.M-M, E.V-R and N.A) and a post-doctoral graduate program grant (Juan de la Cierva to V.C and postdoc-UJI to A.M-P and M-Á.P-G). Author E.B was funded by a post-doctoral grant from the "Generalitat Valenciana (2018 APOSTD)" and the "European Social Fund (Investing in your future)."

\section{Compliance with ethical standards}

Conflict of Interest The authors declare that they have no conflict of interest.

\section{References}

Adelstein, J. S., Shehzad, Z., Mennes, M., DeYoung, C. G., Zuo, X.-N., Kelly, C., Margulies, D. S., Bloomfield, A., Gray, J. R., Castellanos, F. X., \& Milham, M. P. (2011). Personality Is Reflected in the Brain's Intrinsic Functional Architecture. PLoS ONE, 6(11), e27633. https://doi.org/10.1371/journal.pone.0027633

Adrián-Ventura, J., Costumero, V., Parcet, M. A., \& Ávila, C. (2019). Linking personality and brain anatomy: a structural MRI approach to Reinforcement Sensitivity Theory. Social Cognitive and Affective Neuroscience, 14(3), 329-338. https://doi.org/10.1093/scan/nsz011

Aghajani, M., Veer, I. M., van Tol, M.-J., Aleman, A., van Buchem, M. A., Veltman, D. J., Rombouts, S. A. R. B., \& van der Wee, N. J. (2014). Neuroticism and extraversion are associated with amygdala resting-state functional connectivity. Cognitive, Affective \& Behavioral Neuroscience, 14(2), 836-848. https://doi.org/10.3758/ s13415-013-0224-0

Aluja, A., \& Blanch, A. (2011). Neuropsychological Behavioral Inhibition System (BIS) and Behavioral Approach System (BAS) assessment: a shortened Sensitivity to Punishment and Sensitivity to Reward Questionnaire version (SPSRQ-20). Journal of Personality Assessment, 93(6), 628-636. https://doi.org/10.1080/00223891. 2011.608760

Ashburner, J. (2007). A fast diffeomorphic image registration algorithm. NeuroImage, 38(1), 95-113. https://doi.org/10.1016/J. NEUROIMAGE.2007.07.007

Ávila, C., \& Torrubia, R. (2008). Performance and conditioning studies. In P. J. Corr (Ed.), The reinforcement sensitivity theory of personality (pp. 228-260). Cambridge University Press.

Baeken, C., Marinazzo, D., Van Schuerbeek, P., Wu, G. R., De Mey, J., Luypaert, R., \& De Raedt, R. (2014). Left and right amygdala Mediofrontal cortical functional connectivity is differentially modulated by harm avoidance. PLOS ONE, 9(4), e95740. https://doi.org/ 10.1371/journal.pone.0095740 
Barrós-Loscertales, A., Meseguer, V., Sanjuán, A., Belloch, V., Parcet, M. A., Torrubia, R., \& Avila, C. (2006). Behavioral Inhibition System activity is associated with increased amygdala and hippocampal gray matter volume: A voxel-based morphometry study. NeuroImage, 33(3), 1011-1015. https://doi.org/10.1016/j. neuroimage.2006.07.025

Ben-Simon, E., Podlipsky, I., Arieli, A., Zhdanov, A., \& Hendler, T. (2008). Never resting brain: simultaneous representation of two alpha related processes in humans. PloS ONE, 3(12), e3984. https:// doi.org/10.1371/journal.pone.0003984

Benjamini, Y., \& Hochberg, Y. (1995). Controlling the False Discovery Rate: A Practical and Powerful Approach to Multiple Testing. Journal of the Royal Statistical Society. Series B (Methodological), 57(1), 289-300. https://doi.org/10.2307/2346101

Berger, H. (1929). Über das Elektrenkephalogramm des Menschen. Archiv Für Psychiatrie Und Nervenkrankheiten, 87(1), 527-570. https://doi.org/10.1007/BF01797193

Bijttebier, P., Beck, I., Claes, L., \& Vandereycken, W. (2009). Gray's Reinforcement Sensitivity Theory as a framework for research on personality-psychopathology associations. Clinical Psychology Review, 29(5), 421-430. https://doi.org/10.1016/j.cpr.2009.04.002

Buckholtz, J. W., Callicott, J. H., Kolachana, B., Hariri, A. R., Goldberg, T. E., Genderson, M., Egan, M. F., Mattay, V. S., Weinberger, D. R., \& Meyer-Lindenberg, A. (2008). Genetic variation in MAOA modulates ventromedial prefrontal circuitry mediating individual differences in human personality. Molecular Psychiatry, 13(3), 313-324. https://doi.org/10.1038/sj.mp.4002020

Burkhouse, K. L., Jacobs, R. H., Peters, A. T., Ajilore, O., Watkins, E. R., \& Langenecker, S. A. (2017). Neural correlates of rumination in adolescents with remitted major depressive disorder and healthy controls. Cognitive, Affective and Behavioral Neuroscience, 17(2), 394-405. https://doi.org/10.3758/s13415-016-0486-4

Caseras, X., Àvila, C., \& Torrubia, R. (2003). The measurement of individual differences in Behavioural Inhibition and Behavioural Activation Systems: a comparison of personality scales. Personality and Individual Differences, 34(6), 999-1013. https:// doi.org/10.1016/S0191-8869(02)00084-3

Cavanna, A. E., \& Trimble, M. R. (2006). The precuneus: A review of its functional anatomy and behavioural correlates. Brain, 129(3), 564 583. https://doi.org/10.1093/brain/aw1004

Cherbuin, N., Windsor, T. D., Anstey, K. J., Maller, J. J., Meslin, C., \& Sachdev, P. S. (2008). Hippocampal volume is positively associated with behavioural inhibition (BIS) in a large community-based sample of mid-life adults: the PATH through life study. Social Cognitive and Affective Neuroscience, 3(3), 262-269. https://doi.org/10.1093/ scan/nsn018

Corbetta, M., Patel, G., \& Shulman, G. L. (2008). The reorienting system of the human brain: from environment to theory of mind. Neuron, 58(3), 306-324. https://doi.org/10.1016/j.neuron.2008.04.017

Corbetta, M., \& Shulman, G. L. (2002). Control of goal-directed and stimulus-driven attention in the brain. Nature Reviews. Neuroscience, 3(3), 201-215. https://doi.org/10.1038/nrn755

Corr, P. J. (2004). Reinforcement sensitivity theory and personality. Neuroscience and Biobehavioral Reviews, 28(3), 317-332. https:// doi.org/10.1016/j.neubiorev.2004.01.005

Costumero, V., Bueichekú, E., Adrián-Ventura, J., \& Ávila, C. (2020). Opening or closing eyes at rest modulates the functional connectivity of V1 with default and salience networks. Scientific Reports, 10(1), 9137. https://doi.org/10.1038/s41598-020-66100-y

da Silva, P. H. R., Rondinoni, C., \& Leoni, R. F. (2020). Non-classical behavior of the default mode network regions during an information processing task. Brain Structure and Function, 225(8), 2553-2562. https://doi.org/10.1007/s00429-020-02143-1

Ding, X., Tang, Y. Y., Deng, Y., Tang, R., \& Posner, M. I. (2015). Mood and personality predict improvement in creativity due to meditation training. Learning and Individual Differences, 37, 217-221. https:// doi.org/10.1016/j.lindif.2014.11.019

Duncan, N. W., Wiebking, C., Tiret, B., Marjańska, M., Hayes, D. J., Lyttleton, O., Doyon, J., \& Northoff, G. (2013). Glutamate Concentration in the Medial Prefrontal Cortex Predicts RestingState Cortical-Subcortical Functional Connectivity in Humans. PLoS ONE, 8(4), e60312. https://doi.org/10.1371/journal.pone. 0060312

Edlow, B. L., Takahashi, E., Wu, O., Benner, T., Dai, G., Bu, L., Grant, P. E., Greer, D. M., Greenberg, S. M., Kinney, H. C., \& Folkerth, R. D. (2012). Neuroanatomic connectivity of the human ascending arousal system critical to consciousness and its disorders. Journal of Neuropathology and Experimental Neurology, 71(6), 531-546. https://doi.org/10.1097/NEN.0b013e3182588293

Fanselow, M. S. (1991). The Midbrain Periaqueductal Gray as a Coordinator of Action in Response to Fear and Anxiety. In A. Depaulis \& R. Bandler (Eds.), The Midbrain Periaqueductal Gray Matter (pp. 151-173). Plenum Press. https://doi.org/10.1007/978-14615-3302-3_10

Friston, K. J., Williams, S., Howard, R., Frackowiak, R. S. J., \& Turner, R. (1996). Movement-Related effects in fMRI time-series. Magnetic Resonance in Medicine, 35(3), 346-355. https://doi.org/10.1002/ mrm. 1910350312

Fuentes, P., Barrós-Loscertales, A., Bustamante, J. C., Rosell, P., Costumero, V., \& Ávila, C. (2012). Individual differences in the Behavioral Inhibition System are associated with orbitofrontal cortex and precuneus gray matter volume. Cognitive, Affective \& Behavioral Neuroscience, 12(3), 491-498. https://doi.org/10.3758/ s13415-012-0099-5

Gentili, C., Cristea, I. A., Ricciardi, E., Vanello, N., Popita, C., David, D., \& Pietrini, P. (2017). Not in one metric: Neuroticism modulates different resting state metrics within distinctive brain regions. Behavioural Brain Research, 327, 34 43. https://doi.org/10.1016/ j.bbr.2017.03.031

Gray, J. A., \& McNaughton, N. (2000). The neuropsychology of anxiety: An enquiry into the functions of the septo-hippocampal system (2nd ed.). Oxford University Press.

Hahn, T., Dresler, T., Plichta, M. M., Ehlis, A.-C., Ernst, L. H., Markulin, F., Polak, T., Blaimer, M., Deckert, J., Lesch, K.-P., Jakob, P. M., \& Fallgatter, A. J. (2010). Functional amygdala-hippocampus connectivity during anticipation of aversive events is associated with Gray's trait "sensitivity to punishment". Biological Psychiatry, 68(5), 459-464. https://doi.org/10.1016/j.biopsych.2010.04.033

Holmes, A. J., Lee, P. H., Hollinshead, M. O., Bakst, L., Roffman, J. L., Smoller, J. W., \& Buckner, R. L. (2012). Individual differences in amygdala-medial prefrontal anatomy link negative affect, impaired social functioning, and polygenic depression risk. The Journal of Neuroscience, 32(50), 18087-18100. https://doi.org/10.1523/ JNEUROSCI.2531-12.2012

Hüfner, K., Stephan, T., Flanagin, V. L., Deutschländer, A., Stein, A., Kalla, R., Dera, T., Fesl, G., Jahn, K., Strupp, M., \& Brandt, T. (2009). Differential effects of eyes open or closed in darkness on brain activation patterns in blind subjects. Neuroscience Letters, 466(1), 30-34. https://doi.org/10.1016/j.neulet.2009.09.010

Huggins, A. A., Belleau, E. L., Miskovich, T. A., Pedersen, W. S., \& Larson, C. L. (2018). Moderating effects of harm avoidance on resting-state functional connectivity of the anterior insula. Frontiers in Human Neuroscience, 12, 447. https://doi.org/10. 3389/fnhum.2018.00447

Jao, T., Vértes, P. E., Alexander-Bloch, A. F., Tang, I.-N., Yu, Y.-C., Chen, J.-H., \& Bullmore, E. T. (2013). Volitional eyes opening perturbs brain dynamics and functional connectivity regardless of light input. NeuroImage, 69, 21-34. https://doi.org/10.1016/j. neuroimage.2012.12.007

Kennis, M., Rademaker, A. R., \& Geuze, E. (2013). Neural correlates of personality: an integrative review. Neuroscience and Biobehavioral 
Reviews, 37(1), 73-95. https://doi.org/10.1016/j.neubiorev.2012.10. 012

Kim, M. J., Gee, D. G., Loucks, R. A., Davis, F. C., \& Whalen, P. J. (2011). Anxiety dissociates dorsal and ventral medial prefrontal cortex functional connectivity with the amygdala at rest. Cerebral Cortex, 21(7), 1667-1673. https://doi.org/10.1093/cercor/bhq237

Konareva, I. N. (2011a). Modulation of high-frequency EEG rhythms under conditions of the activation reaction: Dependence on psychological characteristics of personality. Neurophysiology, 43(1), 4252. https://doi.org/10.1007/s11062-011-9184-6

Konareva, I. N. (2011b). Modulation of low-frequency EEG rhythms under conditions of an activation reaction: Dependence on psychological characteristics of personality. Neurophysiology, 42(6), 434 445. https://doi.org/10.1007/s11062-011-9179-3

Kruschwitz, J. D., Walter, M., Varikuti, D., Jensen, J., Plichta, M. M., Haddad, L., Grimm, O., Mohnke, S., Pöhland, L., Schott, B., Wold, A., Mühleisen, T. W., Heinz, A., Erk, S., Romanczuk-Seiferth, N., Witt, S. H., Nöthen, M. M., Rietschel, M., Meyer-Lindenberg, A., \& Walter, H. (2014). 5-HTTLPR/rs25531 polymorphism and neuroticism are linked by resting state functional connectivity of amygdala and fusiform gyrus. Brain Structure and Function, 220(4), 23732385. https://doi.org/10.1007/s00429-014-0782-0

Leen-Feldner, E. W., Zvolensky, M. J., Feldner, M. T., \& Lejuez, C. W. (2004). Behavioral inhibition: Relation to negative emotion regulation and reactivity. Personality and Individual Differences, 36(6), 1235-1247. https://doi.org/10.1016/S0191-8869(02)00113-7

Levita, L., Bois, C., Healey, A., Smyllie, E., Papakonstantinou, E., Hartley, T., \& Lever, C. (2014). The Behavioural Inhibition System, anxiety and hippocampal volume in a non-clinical population. Biology of Mood \& Anxiety Disorders, 4(1), 4. https://doi.org/ 10.1186/2045-5380-4-4

Li, W., Cui, H., Zhu, Z., Kong, L., Guo, Q., Zhu, Y., Hu, Q., Zhang, L., Li, H., Li, Q., Jiang, J., Meyers, J., Li, J., Wang, J., Yang, Z., \& Li, C. (2016). Aberrant Functional Connectivity between the Amygdala and the Temporal Pole in Drug-Free Generalized Anxiety Disorder. Frontiers in Human Neuroscience, 10, 549. https://doi.org/10.3389/ fnhum.2016.00549

Li, Y., Qin, W., Jiang, T., Zhang, Y., \& Yu, C. (2012). Sex-dependent correlations between the personality dimension of harm avoidance and the resting-state functional connectivity of amygdala subregions. PLoS ONE, 7(4), e35925. https://doi.org/10.1371/journal. pone. 0035925

Liang, B., Zhang, D., Wen, X., Xu, P., Peng, X., Huang, X., Liu, M., \& Huang, R. (2014). Brain spontaneous fluctuations in sensorimotor regions were directly related to eyes open and eyes closed: evidences from a machine learning approach. Frontiers in Human Neuroscience, 8, 645. https://doi.org/10.3389/fnhum.2014.00645

Linnman, C., Moulton, E. A., Barmettler, G., Becerra, L., \& Borsook, D. (2012). Neuroimaging of the periaqueductal gray: State of the field. NeuroImage, 60(1), 505-522. https://doi.org/10.1016/j.neuroimage. 2011.11.095

Liu, D., Dong, Z., Zuo, X., Wang, J., \& Zang, Y. (2013). Eyes-open/eyesclosed dataset sharing for reproducibility evaluation of resting state fMRI data analysis methods. Neuroinformatics, 11(4), 469-476. https://doi.org/10.1007/s12021-013-9187-0

Mandell, D., Siegle, G. J., Shutt, L., Feldmiller, J., \& Thase, M. E. (2014). Neural substrates of trait ruminations in depression. Journal of Abnormal Psychology, 123(1), 35-48. https://doi.org/10.1037/ a0035834

Markett, S., Weber, B., Voigt, G., Montag, C., Felten, A., Elger, C., \& Reuter, M. (2013). Intrinsic connectivity networks and personality: The temperament dimension harm avoidance moderates functional connectivity in the resting brain. Neuroscience, 240, 98-105. https:// doi.org/10.1016/j.neuroscience.2013.02.056

Marx, E., Deutschländer, A., Stephan, T., Dieterich, M., Wiesmann, M., \& Brandt, T. (2004). Eyes open and eyes closed as rest conditions: impact on brain activation patterns. NeuroImage, 21(4), 1818-1824. https://doi.org/10.1016/j.neuroimage.2003.12.026

Marx, E., Stephan, T., Nolte, A., Deutschländer, A., Seelos, K. C., Dieterich, M., \& Brandt, T. (2003). Eye closure in darkness animates sensory systems. NeuroImage, 19(3), 924-934. https://doi. org/10.1016/s1053-8119(03)00150-2

McNaughton, N., \& Corr, P. J. (2004). A two-dimensional neuropsychology of defense: fear/anxiety and defensive distance. Neuroscience and Biobehavioral Reviews, 28(3), 285-305. https://doi.org/10. 1016/j.neubiorev.2004.03.005

McNaughton, N., \& Corr, P. J. (2008). The neuropsychology of fear and anxiety: a foundation for Reinforcement Sensitivity Theory. In P. J. Corr (Ed.), The reinforcement sensitivity theory of personality (pp. 44-94). Cambridge University Press.

Mellet, E., Petit, L., Mazoyer, B., Denis, M., \& Tzourio, N. (1998). Reopening the mental imagery debate: lessons from functional anatomy. NeuroImage, 8(2), 129-139. https://doi.org/10.1006/nimg. 1998.0355

Meylakh, N., \& Henderson, L. A. (2016). Dorsal raphe nucleus and harm avoidance: A resting-state investigation. Cognitive, Affective and Behavioral Neuroscience, 16(3), 561-569. https://doi.org/10.3758/ s13415-016-0415-6

Modi, S., Kumar, M., Kumar, P., \& Khushu, S. (2015). Aberrant functional connectivity of resting state networks associated with trait anxiety. Psychiatry Research: Neuroimaging, 234(1), 25-34. https://doi.org/10.1016/j.pscychresns.2015.07.006

Oldfield, R. C. C. (1971). The assessment and analysis of handedness: The Edinburgh inventory. Neuropsychologia, 9(1), 97-113. https:// doi.org/10.1016/0028-3932(71)90067-4

Pang, J., Tang, X., Li, H., Hu, Q., Cui, H., Zhang, L., Li, W., Zhu, Z., Wang, J., \& Li, C. (2019). Altered Interoceptive Processing in Generalized Anxiety Disorder-A Heartbeat-Evoked Potential Research. Frontiers in Psychiatry, 10, 616. https://doi.org/10. 3389/fpsyt.2019.00616

Pang, Y., Cui, Q., Wang, Y., Chen, Y., Wang, X., Han, S., Zhang, Z., Lu, G., \& Chen, H. (2016). Extraversion and neuroticism related to the resting-state effective connectivity of amygdala. Scientific Reports, 6(1), 35484. https://doi.org/10.1038/srep35484

Power, J. D., Barnes, K. A., Snyder, A. Z., Schlaggar, B. L., \& Petersen, S. E. (2012). Spurious but systematic correlations in functional connectivity MRI networks arise from subject motion. NeuroImage, 59(3), 2142-2154. https://doi.org/10.1016/J.NEUROIMAGE. 2011.10.018

Ptak, R., Schnider, A., \& Fellrath, J. (2017). The Dorsal Frontoparietal Network: A Core System for Emulated Action. Trends in Cognitive Sciences, 21(8), 589-599. https://doi.org/10.1016/j.tics.2017.05. 002

Raichle, M. E., MacLeod, A. M., Snyder, A. Z., Powers, W. J., Gusnard, D. A., \& Shulman, G. L. (2001). A default mode of brain function. Proceedings of the National Academy of Sciences of the United States of America, 98(2), 676-682. https://doi.org/10.1073/pnas. 98.2.676

Song, X., Zhou, S., Zhang, Y., Liu, Y., Zhu, H., \& Gao, J.-H. (2015). Frequency-Dependent Modulation of Regional Synchrony in the Human Brain by Eyes Open and Eyes Closed Resting-States. PloS ONE, 10(11), e0141507. https://doi.org/10.1371/journal.pone. 0141507

Tomasino, B., \& Gremese, M. (2016). Effects of Stimulus Type and Strategy on Mental Rotation Network: An Activation Likelihood Estimation Meta-Analysis. Frontiers in Human Neuroscience, 9, 693. https://doi.org/10.3389/fnhum.2015.00693

Torrubia, R., Ávila, C., Moltó, J., \& Caseras, X. (2001). The Sensitivity to Punishment and Sensitivity to Reward Questionnaire (SPSRQ) as a measure of Gray's anxiety and impulsivity dimensions. Personality and Individual Differences, 31(6), 837-862. https://doi.org/10. 1016/S0191-8869(00)00183-5 
Tzourio-Mazoyer, N., Landeau, B., Papathanassiou, D., Crivello, F., Etard, O., Delcroix, N., Mazoyer, B., \& Joliot, M. (2002). Automated Anatomical Labeling of Activations in SPM Using a Macroscopic Anatomical Parcellation of the MNI MRI SingleSubject Brain. NeuroImage, 15(1), 273-289. https://doi.org/10. 1006/nimg.2001.0978

Wang, X.-H., Li, L., Xu, T., \& Ding, Z. (2015). Investigating the Temporal Patterns within and between Intrinsic Connectivity Networks under Eyes-Open and Eyes-Closed Resting States: A Dynamical Functional Connectivity Study Based on Phase Synchronization. PloS ONE, 10(10), e0140300. https://doi.org/10. 1371/journal.pone. 0140300

Weber-Goericke, F., \& Muehlhan, M. (2019). A quantitative metaanalysis of fMRI studies investigating emotional processing in excessive worriers: Application of activation likelihood estimation analysis. Journal of Affective Disorders, 243, 348-359. https://doi. org/10.1016/j.jad.2018.09.049

Wei, J., Chen, T., Li, C., Liu, G., Qiu, J., \& Wei, D. (2018). Eyes-Open and Eyes-Closed Resting States With Opposite Brain Activity in Sensorimotor and Occipital Regions: Multidimensional Evidences From Machine Learning Perspective. Frontiers in Human Neuroscience, 12, 422. https://doi.org/10.3389/fnhum.2018.00422

Wiesmann, M., Kopietz, R., Albrecht, J., Linn, J., Reime, U., Kara, E., Pollatos, O., Sakar, V., Anzinger, A., Fesl, G., Brückmann, H., Kobal, G., \& Stephan, T. (2006). Eye closure in darkness animates olfactory and gustatory cortical areas. NeuroImage, 32(1), 293-300. https://doi.org/10.1016/j.neuroimage.2006.03.022

Xu, P., Huang, R., Wang, J., Van Dam, N. T., Xie, T., Dong, Z., Chen, C., Gu, R., Zang, Y.-F., He, Y., Fan, J., \& Luo, Y. (2014). Different topological organization of human brain functional networks with eyes open versus eyes closed. NeuroImage, 90, 246-255. https://doi. org/10.1016/j.neuroimage.2013.12.060

Yan, C.-G., Wang, X.-D., Zuo, X.-N., \& Zang, Y.-F. (2016). DPABI: Data Processing \& Analysis for (Resting-State) Brain Imaging. Neuroinformatics, 14(3), 339-351. https://doi.org/10.1007/s12021016-9299-4

Zhang, D., Liang, B., Wu, X., Wang, Z., Xu, P., Chang, S., Liu, B., Liu, M., \& Huang, R. (2015). Directionality of large-scale resting-state brain networks during eyes open and eyes closed conditions. Frontiers in Human Neuroscience, 9, 81. https://doi.org/10.3389/ fnhum.2015.00081

Zhang, S., \& Li, C.-S. R. (2012). Functional connectivity mapping of the human precuneus by resting state fMRI. NeuroImage, 59(4), 35483562. https://doi.org/10.1016/j.neuroimage.2011.11.023

Open Practices Statement

The data that support the findings of this study are available from the corresponding author upon reasonable request. None of the experiments from this article was formally preregistered.

Publisher's note Springer Nature remains neutral with regard to jurisdictional claims in published maps and institutional affiliations. 